\title{
Correction to: Subgroups of patients with young-onset type 2 diabetes in India reveal insulin deficiency as a major driver
}

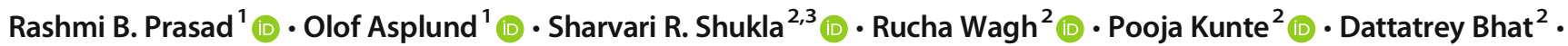

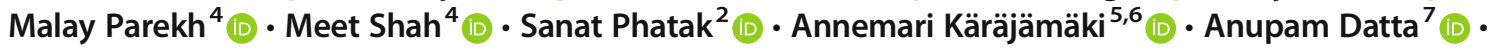 \\ Sanjeeb Kakati ${ }^{7}$ (D) Tiinamaija Tuomi $^{1,8,9,10}$ (D) Banshi Saboo $^{4}$ (D) Emma Ahlqvist $^{1}$ (D) $\cdot$ Leif Groop $^{1,10}$ (D) • \\ Chittaranjan S. Yajnik ${ }^{2}$ (I)
}

Published online: 22 November 2021

(C) Springer-Verlag GmbH Germany, part of Springer Nature 2021

\section{Correction to: Diabetologia.}

https://doi.org/10.1007/s00125-021-05543-y

The name Malay Parekh was spelt incorrectly in the author list. The original article has been corrected.

\section{Rashmi B. Prasad} rashmi.prasad@med.lu.se

$\triangle$ Chittaranjan S. Yajnik csyajnik@gmail.com

1 Department of Clinical Sciences, Diabetes and Endocrinology, CRC, Lund University, Malmö, Sweden

2 Diabetes Unit, Kamalnayan Bajaj Diabetology Research Centre, King Edward Memorial Hospital and Research Centre, Pune, India

3 Symbiosis Statistical Institute, Symbiosis International University, Pune, India

4 Dia Care - Diabetes Hormone Clinic, Ahmedabad, India
5 Department of Primary Health Care, Vaasa Central Hospital, Vaasa, Finland

6 Diabetes Center, Vaasa Health Care Center, Vaasa, Finland

7 Assam Medical College and Hospital, Dibrugarh, India

8 Abdominal Center, Endocrinology, Helsinki University Central Hospital, Research Program for Clinical and Molecular Metabolism, University of Helsinki, Helsinki, Finland

$9 \quad$ Folkhälsan Research Center, Helsinki, Finland

10 Institute for Molecular Medicine Finland FIMM, Helsinki University, 00290 Helsinki, Finland 